\title{
Application of the Kurtosis Metric to the Assessment of Hearing Loss Associated with Occupational Noise Exposure
}

\author{
Wei Qiu ${ }^{1, *}$; Meibian Zhang²; Weijiang $\mathrm{Hu}^{3}$; Xin $\mathrm{Sun}^{3}$
}

It is well known that high-level noise exposure can lead to hearing loss. Noise-induced hearing loss (NIHL) continues to be one of the major occupational health hazards. An underlying assumption in current noise standards, e.g., ISO 1999:2013 (1), is that hearing loss is related to the total energy of the exposure. Thus, the risk of NIHL can be predicted according to the ISO 1999 prediction model. Unfortunately, the validity of the prediction model to correctly predict the NIHL for all types of noise exposure is still under question, especially for complex noise of impulsive character. The basis of current noise guidelines is the equal-energy hypothesis (EEH) approach, i.e., equivalent effects on hearing for a 3-dB increase or decrease in exposure intensity with a halving or doubling of the exposure duration, respectively. This approach is generally considered appropriate for steady-state noise but not for complex noise.

Steady-state continuous noise exposure has a Gaussian amplitude distribution. Therefore, the temporal characteristics of steady-state noise do not change over time. However, noise exposures often vary in the temporal pattern in many work environments. A complex noise is a non-Gaussian noise consisting of a Gaussian background noise punctuated by a temporally complex series of randomly occurring highlevel noise transients. These transients can be brief high-level noise bursts or impacts. Industrial workers are often exposed to complex noise environments. Noises of the same or similar acoustic energies and spectra can have very different effects on hearing because of their different temporal structures.

The fundamental problem with current noise standards, e.g., ISO 1999 (1), is their reliance on an acoustic energy metric to quantify noise exposure. An acoustic energy metric completely ignores the effects of the temporal characteristics of noise exposure known to be important in affecting complex noise-induced hearing loss. Many published papers have shown that exposure to complex noise produces more hearing loss and sensory cell loss than an equivalent energy exposure to steady-state noise does in both animal and human models (2-5). It is reasonable that a metric that would incorporate and reflect the temporal structure of exposure might be a useful adjunct to the equivalent sound pressure level $\left(\mathrm{L}_{\mathrm{eq}}\right)$ metric. One such metric is the kurtosis of a noise exposure $(3,5)$. Kurtosis $(\beta)$ is a statistical measure of extreme values or outliers of a distribution. Kurtosis can be used to describe the amplitude "peakedness" of noise waveforms. It is worth mentioning that the Gaussian distribution has a kurtosis of 3. A complex non-Gaussian noise, $\beta>3$, can be effectively modeled as a combination of Gaussian noise, $\quad \beta=3$, with various high-level transients superimposed.

\section{THE KURTOSIS METRIC AS AN ADJUNCT TO ENERGY IN THE EVALUATION OF NIHL}

A team of researchers conducted a series of animal (chinchilla) experiments to investigate whether kurtosis can differentiate the NIHL for a fixed Leq $(2-3,6-7)$. The results showed that: i) non-Gaussian noise exposures are more hazardous than continuous Gaussian noise exposures of equivalent energy, and noise kurtosis explains much of this increased hazard; ii) NIHL increase as kurtosis increases for a fixed $\mathrm{L}_{\text {eq }}$; and iii) both kurtosis and energy $\left(\mathrm{L}_{\mathrm{eq}}\right)$ are necessary to evaluate the hazard posed to hearing by complex noise exposure.

Recently, large-scale epidemiological studies have been carried out in Chinese industries, and the data have been fully analyzed (5). The results were in general agreement with the above-mentioned animal models showing that: i) an acoustic energy metric is necessary but not sufficient to evaluate the hazard of noise to hearing; ii) the temporal distribution of energy of noise (i.e., kurtosis) is an important factor in assessing noise-induced hearing loss; iii) for a fixed energy level, the noise-induced hearing loss increased as the kurtosis of the noise increased; and iv) non- 
Gaussian complex noises are more hazardous than Gaussian noise exposures of equivalent energy, and the hazard is identified by the kurtosis value of the noise.

\section{CALCULATION METHOD AND ADJUSTMENT MODELS USING NOISE KURTOSIS}

\section{Calculating the Kurtosis of Noise Exposure}

If, based on existing data, one accepts the proposition that kurtosis should be routinely measured in all industrial noise exposures, then the question of how best to measure the kurtosis metric should be considered. The kurtosis of the noise sample is dependent upon not just the probability of a high amplitude event to occur within the sample window, but also the length of the sample window. Recently, two algorithms were designed by Tian and colleagues (8) to investigate the correlation between window duration for kurtosis computation and the accuracy of NIHL prediction using a Chinese industrial database. They found that 60 seconds is the optimal window length for kurtosis calculation. Therefore, the kurtosis of noise exposure should be computed over consecutive 60-second time windows without overlap over the shift-long noise record using a sampling rate of 48 $\mathrm{kHz}$. The mean of the measured kurtosis values is calculated and used as the kurtosis metric.

\section{The Kurtosis Adjustment Models}

So far, two kurtosis adjustment models have been proposed as follows:

\section{Model 1 - kurtosis adjustment through exposure time:}

This model was used in Zhao et al. (4) and Xie et al. (9). The adjustment formula is listed below:

$$
C N E_{\text {kurtosis-adjusted }}=L_{\text {Aeq. } 8 h}+\frac{\ln (\beta)+1.9}{\log (2)} \log (T)
$$

This form was chosen for calculating the kurtosisadjusted cumulative noise exposure (CNE) because Gaussian noise has a kurtosis of $\beta=3$, and the term $[(\ln (\beta)+1.9) / \log (2)]$ is close to 10 . Thus, for Gaussian noise, the kurtosis-adjusted CNE equals the unadjusted CNE. According to Equation (1), for a fixed $\mathrm{L}_{\text {Aeq,8h, }}$, the kurtosis adjusted $\mathrm{CNE}$ of the nonGaussian noise $(\beta>3)$ is larger than that of the Gaussian noise $(\beta=3)$, which is equivalent to prolonging the noise exposure duration.

Model 2 - kurtosis adjustment through $L_{\text {Aeq }}$

Goley et al. (10) presented another way to use kurtosis in NIHL evaluation. Goley and colleagues proposed a scheme that uses kurtosis to adjust the Aweighted equivalent sound pressure level $\left(L_{\text {Aeq }}\right)$ directly. The basic form of the kurtosis-adjusted $L_{\text {Aeq }}^{\prime}$ was determined as follows:

$$
L_{A e q}^{\prime}=L_{A e q}+\lambda \log _{10} \frac{\beta_{N}}{\beta_{G}}
$$

where $\lambda$ is a positive constant to be determined from the dose-response correlation study, $\beta_{\mathrm{N}}$ is the kurtosis of the noise, and $\beta_{G}=3$ is the kurtosis of the Gaussian noise. Taking noise-induced permanent threshold shift as the dependent variable and $L_{\text {Acq }}$ and $\log \left(\beta_{N} / 3\right)$ as independent variables, the coefficient $\lambda$ was calculated by multiple linear regression model. Based on the animal (chinchilla) model, Goley obtained $\lambda=4.02$. If the model is applied to humans, the value needs to be re-estimated using human data. Using Goley's model is equivalent to adding an increment determined by the second term of the formula to the resulting total sound pressure level.

\section{APPLICATION OF THE KURTOSIS METRIC IN INDUSTRIAL SETTINGS}

Zhao et al. (4) published the first preliminary study of using kurtosis in industrial settings. Using kurtosisadjustment Equation (1), they evaluated the prevalence of adjusted high-frequency NIHL (AHFNIHL) in workers exposed to Gaussian $(\mathrm{N}=163)$ and nonGaussian noise $(\mathrm{N}=32)$. The prevalence of AHFNIHL in workers was defined as having one or more hearing thresholds, in either ear, at 3,4,6 kHz equal to or higher than $30 \mathrm{~dB}$ HL. A dose-response relation for the Gaussian and non-Gaussian noise-exposed groups was then constructed. By introducing the kurtosis variable into the temporal component of the $\mathrm{CNE}$ calculation, the two dose-response curves were made to overlap, essentially yielding an equivalent noise-induced effect (i.e., AHFNIHL) for the two study groups. Thus, the kurtosis metric was used to quantify the deviation of the non-Gaussian noise environment from the Gaussian noise environment. The results showed that the kurtosis metric could more accurately assess the risk of developing AHFNIHL in workers exposed to high-level Gaussian and non-Gaussian noise. Xie et al. (9) conducted another study, including 178 workers exposed to complex non-Gaussian noise and 163 
participants exposed to Gaussian noise. The result showed that using a kurtosis-adjusted CNE, the AHFNIHL-CNE relationship curves of the complex noise and $G$ noise overlapped. This result supported the work from Zhao et al. (4).

Fuente et al. (11) conducted a pilot study in China to determine the synergistic effect of solvents combined with non-Gaussian noise in humans. The kurtosis metric was calculated to quantify the temporal structure of the noise. The CNE was used to quantify the noise exposure for each participant. This index was also adjusted by the kurtosis metric using Equation (1). The change in slope for the relationship between CNE and kurtosis-adjusted CNE and hearing thresholds in each exposure group was analyzed to determine whether there was a significant effect in the interaction. The results showed that the interaction term between CNE and exposure group on hearing thresholds (1-8 $\mathrm{kHz}$ ) was not statistically significant. However, the interaction term between kurtosis-adjusted $\mathrm{CNE}$ and the exposure group showed a statistically significant effect at $6 \mathrm{kHz}$. Therefore, an interaction between noise and solvent exposure on the pure-tone threshold at $6 \mathrm{kHz}$ was observed only when the temporal structure of noise was considered. This pilot study provides evidence that using a kurtosis metric that incorporates the impulsiveness of noise combined with solvent exposure can allow the detection of their effects on the hearing threshold.

There is a growing interest in using kurtosis as a metric for examining the effects of anthropogenic noise on the hearing of marine mammals, fishes, and other marine organisms. Kurtosis was recommended as one of the marine mammal noise exposure criteria, and kurtosis was used as one of the quantitative metrics to describe the underwater soundscape. Muller et al. (12) demonstrated how the kurtosis of underwater sounds could be measured unambiguously. They provided practical formulas for evaluating the kurtosis of impulsive sounds.

\section{THE APPLICATION PROSPECT OF KURTOSIS IN NIHL EVALUATION}

Researchers have long been aware that under the same energy and spectrum conditions, complex noise with impulsive components is more hazardous to hearing than steady-state noise. Therefore, using the equal energy rule is not sufficiently protective against noise exposures other than continuous or Gaussian noise. The 1971 version of the ISO 1999 document mentioned an increase of $10 \mathrm{~dB}$ for noise with impulsive components, while the 1990 version mentioned a correction of $5 \mathrm{~dB}$. However, since there is no specific quantitative standard, such a correction method is arbitrary. The above studies have shown that kurtosis can effectively evaluate the temporal characteristics of noise exposure for hearing conservation purpose. Correspondingly, animal and epidemiological studies have demonstrated that NIHL increases monotonically with the increase of kurtosis. With the development of the human kurtosis database, the kurtosis-adjustment model can provide us with more accurate NIHL prediction, thus providing a reliable basis for establishing more effective hearing protection programs for the workers exposed to occupational noise.

Funding: National Institute for Occupational Safety and Health, USA (Grant No. 200-2015-M-63857, 200-2016-M-91922); Zhejiang Province Key Research and Development Project, China (Grant No. 2015C03039); Zhejiang Provincial Program for the Cultivation of High-level Innovative Health Talents, China (Grant No, 2016-63-07); Program of Occupational Health Risk Assessment of China NIOHP (Grant No. 131031109000160004); and Occupational Health Standards Preliminary Research Project of China NIOHP (Grant No. 20210102).

doi: $10.46234 / \mathrm{ccdcw} 2021.105$

\# Corresponding author: Wei Qiu, qiuw@plattsburgh.edu.

\begin{abstract}
${ }^{1}$ Auditory Research Laboratory, State University of New York at Plattsburgh, New York, USA; ${ }^{2}$ Zhejiang Provincial Center for Disease Control and Prevention, Hangzhou, Zhejiang, China; ${ }^{3}$ National Institute of Occupational Health and Poison Control, Beijing, China.
\end{abstract}

Submitted: April 13, 2021; Accepted: April 28, 2021

\section{REFERENCES}

1. ISO (International Standard Organization) 1999 (2013). AcousticsDetermination of Occupational Noise Exposure and Estimation NoiseInduced Hearing Impairment. ISO/TC43-Acoustics. Geneva, Switzerland: International Organization for Standardization. https:// www.techstreet.com/standards/bs-iso-1999-2013?product_id=1867062.

2. Lei SF, Ahroon WA, Hamernik RP. The application of frequency and time domain kurtosis to the assessment of hazardous noise exposures. J Acoust Soc Am 1994;96(3):1435 - 44. http://dx.doi.org/10.1121/1. 410287.

3. Qiu W, Hamernik RP, Davis RI. The value of a kurtosis metric in estimating the hazard to hearing of complex industrial noise exposures. J Acoust Soc Am 2013;133(5):2856 - 66. http://dx.doi.org/10.1121/1. 4799813.

4. Zhao YM, Qiu W, Zeng L, Chen SS, Cheng XR, Davis RI, et al. Application of the kurtosis statistic to the evaluation of the risk of hearing loss in workers exposed to high-level complex noise. Ear Hear 
2010;31(4):527 - 32. http://dx.doi.org/10.1097/AUD.0b013e3181d94 e68.

5. Zhang MB, Xie HW, Zhou JN, Sun X, Hu WJ, Zou H, et al. New metrics needed in the evaluation of hearing hazard associated with industrial noise exposure. Ear Hear 2021;42(2):290 - 300. http://dx. doi.org/10.1097/AUD.0000000000000942.

6. Hamernik RP, Qiu W, Davis B. The effects of the amplitude distribution of equal energy exposures on noise-induced hearing loss: the kurtosis metric. J Acoust Soc Am 2003;114(1):386 - 95. http://dx. doi.org/10.1121/1.1582446.

7. Qiu W, Davis B, Hamernik RP. Hearing loss from interrupted, intermittent, and time varying Gaussian noise exposures: the applicability of the equal energy hypothesis. J Acoust Soc Am 2007; 121(3):1613 - 20. http://dx.doi.org/10.1121/1.2434692.

8. Tian Y, Ding WX, Zhang MB, Zhou TS, Li JS, Qiu W. Analysis of correlation between window duration for kurtosis computation and accuracy of noise-induced hearing loss prediction. J Acoust Soc Am
2021;149(4):2367 - 76. http://dx.doi.org/10.1121/10.0003954.

9. Xie HW, Qiu W, Heyer NJ, Zhang MB, Zhang P, Zhao YM, et al. The use of the kurtosis-adjusted cumulative noise exposure metric in evaluating the hearing loss risk for complex noise. Ear Hear 2016;37(3):312 - 23. http://dx.doi.org/10.1097/AUD.000000000000 0251.

10. Goley GS, Song WJ, Kim JH. Kurtosis corrected sound pressure level as a noise metric for risk assessment of occupational noises. J Acoust Soc Am 2011;129(3):1475 - 81. http://dx.doi.org/10.1121/1.3533691.

11. Fuente A, Qiu W, Zhang MB, Xie HW, Kardous CA, Campo P, et al. Use of the kurtosis statistic in an evaluation of the effects of noise and solvent exposures on the hearing thresholds of workers: an exploratory study. J Acoust Soc Am 2018;143(3):1704 - 10. http://dx.doi.org/10. $1121 / 1.5028368$.

12. Müller RAJ, von Benda-Beckmann AM, Halvorsen MB, Ainslie MA. Application of kurtosis to underwater sound. J Acoust Soc Am 2020;148(2):780 - 92. http://dx.doi.org/10.1121/10.0001631. 